\title{
Dificuldades e estratégias de enfrentamento referentes à gestão do trabalho na Estratégia Saúde da Família, na perspectiva dos gestores locais: a experiência dos municípios do Rio de Janeiro (RJ) e Duque de Caxias (RJ)
}

Difficulties and confronting strategies regarding the work management in the Family Health Strategy, in the perspective of local managers: the experience of the municipalities of Rio de Janeiro (RJ) and Duque de Caxias (RJ)

Carinne Magnago', Celia Regina Pierantoni2

RESUMO Objetivou-se identificar as dificuldades e as estratégias de enfrentamento referentes à gestão do trabalho na Estratégia Saúde da Família (ESF). Estudo exploratório, qualitativo, cujos dados foram coletados em 2011, por meio de entrevistas semiestruturadas com gestores dos municípios do Rio de Janeiro (RJ) e Duque de Caxias (RJ), e submetidos à análise de conteúdo. Constituem dificuldades: infraestrutura precária e baixa remuneração. As estratégias para atração e fixação profissional incluem: melhorias em infraestrutura e qualificação. A consolidação da ESF nas metrópoles tem potencialidade de despertar transformações no modelo de atenção que concretizem a saúde como direito social.

PALAVRAS-CHAVE Atenção Primária à Saúde; Estratégia Saúde da Família; Administração de recursos humanos em saúde; Recursos humanos em saúde; Metrópoles.

ABSTRACT It aimed to identify the difficulties and confronting strategies regarding to the labor management in the Family Health Strategy (ESF). Exploratory and qualitative study, whose data were collected in 2011, through semi-structured interviews with managers of the municipalities of Rio de Janeiro (RJ) and Duque de Caxias (RJ), and subjected to content analysis. There are difficulties: precarious infrastructure and low income. Strategies for attracting and professional

1 Universidade do Estado do Rio de Janeiro (Uerj), Instituto de Medicina Social (IMS) - Rio de Janeiro (RJ), Brasil. carinne.mag@gmail.com

2 Universidade do Estado do Rio de Janeiro (Uerj), Instituto de Medicina Social (IMS) - Rio de Janeiro (RJ), Brasil. cpierantoni@gmail.com fixing include: improvements in infrastructure and qualification. The consolidation of the ESF in the metropolis has a potential of awakening changes in the model of care that contribute for the materialization of the health as a social right.

KEYWORDS Primary Health Care; Family Health Strategy; Human resources for health management; Human resources for health; Metropolis. 


\section{Introdução}

A Estratégia Saúde da Família (ESF), anteriormente denominada Programa Saúde da Família (PSF), foi criada em 1994, com o objetivo de reorganizar a Atenção Básica (AB), tanto no que se refere às demandas populacionais quanto no que se refere às práticas de saúde. Esse modelo de atenção derivou do Programa de Agentes Comunitários de Saúde (Pacs), já em prática no País desde 1991, que, por sua vez, foi inspirado em experiências de outros países, como Canadá, Inglaterra e Cuba, que já desenvolviam modelos de assistência com foco na família (VIANA; DAL POZ, 2005).

$\mathrm{O}$ modelo de atenção à saúde proposto pela ESF pretende melhorar o estado de saúde da população priorizando ações de promoção e prevenção, e adotando como unidades de cuidado a família e a comunidade, tornando-se porta de entrada do sistema de saúde e garantindo, por consequência, a hierarquização da assistência.

Nos anos iniciais, o Pacs e a ESF eram prioritários em regiões com maior vulnerabilidade social, de modo que o financiamento federal para tais programas impulsionou a implantação e a expansão de equipes para todo o território nacional, sobretudo nos municípios de baixa densidade demográfica e de pequena ou inexistente rede estruturada de serviços. Nos grandes centros urbanos, por sua vez, a evolução da cobertura se deu de maneira mais lenta porque se deparou com um

elevado grau de exclusão do acesso aos serviços de saúde, agravos de saúde característicos das grandes cidades e metrópoles, e uma oferta consolidada em rede assistencial desarticulada e mal distribuída (ESCOREL ET AL., 2007, P. 165).

A expansão de cobertura nas metrópoles exige maior disponibilidade de recursos financeiros, materiais e humanos, bem como o enfrentamento das resistências corporativas em alterar o processo de trabalho consagrado na lógica de intervenção médica vigente e a dificuldade em superar ou integrar as dimensões de promoção e prevenção às ações de assistência médica (BRASIL, 2002, P. 19).

Constituem-se como dificultadoras do processo de reorganização da rede de saúde das metrópoles algumas particularidades dessas regiões arroladas às demandas da saúde e, por consequência, aos modelos assistenciais. Entre elas, podem ser citadas as alterações demográficas e epidemiológicas imprimidas principalmente pela intensa migração para as grandes cidades, bem como as desigualdades sociais e econômicas que implicam no perfil da demanda e na dificuldade de acesso aos serviços de saúde, associadas à presença de forças paralelas ao Estado legal, como o crime organizado e o tráfico de drogas (CAETANO; DAIN, 2002; CAMPOS; AGUIAR; OLIVEIRA, 2002).

Nos grandes centros, notam-se, ainda, dificuldades relacionadas aos Recursos Humanos em Saúde (RHS), tais como: escassez de profissionais qualificados e com perfil para atuar na $\mathrm{AB}$; modalidades de contrato precárias; e alto índice de rotatividade (NEY; RODRIGUES, 2012; PIERANTONI ET AL., 2010; CAMPOS; AGUIAR; OLIVEIRA, 2002).

Nessa conjuntura, justifica-se o desafio de estudar municípios de grande porte que ainda apresentam dificuldades em consolidar a ESF e fixar profissionais de saúde, não obstante possuírem mercado de trabalho favorável, com maiores possibilidades de progressão acadêmica e profissional, além de ganhos salariais.

Pretende-se contribuir com a elucidação de fatores que dificultam a gestão do trabalho na ESF, nas metrópoles, e apontar estratégias que possam fortalecer a $\mathrm{AB}$ por meio da gestão do trabalho.

Partindo desse contexto, o objetivo deste artigo foi identificar as principais dificuldades e estratégias de enfrentamento referentes à gestão do trabalho percebidas por gestores de RHS. 


\section{Metodologia}

Estudo exploratório de abordagem qualitativa, que teve como cenário de pesquisa os municípios do Rio de Janeiro e de Duque de Caxias, que se localizam na Região Metropolitana I do Estado do Rio de Janeiro e possuem, respectivamente, 6.320 .446 e 855.048 habitantes.

Como técnica de coleta de dados foi utilizada a entrevista semiestruturada, gravada e conduzida com o auxílio de questionário que continha 38 questões divididas em 2 blocos, a saber: (i) Dados de identificação; e (ii) A gestão do trabalho na saúde da família.

Foram entrevistados sete gestores, dentre os quais, quatro do Rio de Janeiro (RJ) e três de Duque de Caxias (RJ). Adotou-se como critério de elegibilidade exercer ou ter exercido cargo de gestão na ESF em um dos municípios estudados; e, como critério de exclusão, não estar disponível no período determinado para a coleta de dados - o segundo semestre de 2011.

As entrevistas foram transcritas na íntegra, submetidas à análise de conteúdo temático e operacionalizadas em três etapas: (i) pré-análise, que consistiu na organização do material a ser analisado mediante leitura e demarcação dos trechos a serem trabalhados; (ii) codificação, classificação e categorização do material; e (iii) interpretação dos resultados (BARDIN, 2002).

A pesquisa foi aprovada pelo Comitê de Ética em Pesquisa do Instituto de Medicina Social, da Universidade do Estado do Rio de Janeiro, sob o registro CAAE 0031.0.259.000-11.

\section{Resultados}

\section{Perfil dos entrevistados}

Foram entrevistados 7 gestores, dos quais a maior parte homens; entre 30 e 40 anos; médicos; com tempo de formação entre 5 e 10 anos; tempo no cargo inferior a 5 anos; pós-graduados em áreas afins à saúde coletiva; com experiência prévia na ESF e em gestão; estatutários; jornada de trabalho de 40 horas com dedicação exclusiva; e com percepção de salários superiores a $\mathrm{R} \$ 6$ mil.

\section{Dificuldades e estratégias de enfren- tamento referentes à gestão do tra- balho na Estratégia Saúde da Família}

Da análise das entrevistas emergiram quatro eixos de discussão, quais sejam: processos educativos; modelo de atenção à saúde; infraestrutura; e vínculos e remuneração, os quais se encontram apresentados segundo o município estudado.

\section{RIO DE JANEIRO}

Dentre as principais dificuldades relacionadas aos recursos humanos, os gestores do Rio de Janeiro (RJ) citam a deficiência no processo de formação, que ainda não prepara o profissional para atuar no âmbito da $A B$, especialmente o médico. E, nesse sentido, o município tem investido não somente na aproximação da rede de saúde com a universidade, mas também na qualificação da sua força de trabalho, disponibilizando cursos técnicos, de especialização e mestrado.

\begin{abstract}
A maior dificuldade hoje talvez seja a fixação do profissional médico, por falta de formação desses profissionais. $E$ aí, a gente está investindo pesadamente em abrir campos de estágios para todas as universidades que tenham interesse. $A$ secretaria está gastando aproximadamente $R \$$ 10 milhões por ano, com cursos, com processos educativos formais.
\end{abstract}

Também como dificuldade no processo de gestão do trabalho, há a percepção de que os profissionais e usuários ainda relutam em compreender e aceitar a mudança do modelo de gestão, de um centrado no médico, com foco na doença e organizado por demanda espontânea, para um que se 
alicerça na equipe multiprofissional e nas ações de caráter de promoção e prevenção de saúde.

Tem sido muito difícil fazer a mudança do modelo [...]. Conciliar isso com a satisfação dos profissionais e com a expectativa da população é um desafio para todos os sistemas do mundo.

No que tange aos obstáculos mais diretamente relacionados à gestão do trabalho, os gestores citaram os vínculos empregatícios e a remuneração praticada pelo município, mas afirmam que o cenário tem apresentado mudanças significativas desde que as equipes de saúde da família passaram a ser geridas pelas Organizações Sociais (OS).

Até 2009, tínhamos profissionais contratados por várias formas, [...] formas mais precárias possíveis. Hoje a gente só tem dois tipos de contratação: estatutário ou funcionário CLT contratado via Organização Social. [...] A gente consegue, através do funcionário CLT, ter uma autonomia um pouco maior de acompanhar o mercado de trabalho e praticar salários iguais ou acima do mercado para poder fixar.

Não obstante, há atritos entre os servidores e os contratados pelas OS, já que se praticam salários e gratificações diferenciadas quando as atividades desenvolvidas são as mesmas.

A gratificação que hoje é paga pelo município ao servidor, comparada à que é paga pela Organização Social, é destoante, [...]de certa forma desestimula um pouco o servidor, principalmente, se você considerar que, desde 2002, esta gratificação existe e [...] permanece com valor inalterado. [...] Uma das maiores dificuldades é tentar criar um ambiente menos conflituoso, menos hostil entre essas duas partes.

A infraestrutura, apesar de referida pelos entrevistados como um fator importante no processo de gestão, já que gera desestímulo aos trabalhadores, está sendo foco de atenção. A prefeitura tem investido na construção de novas unidades e reforma das já existentes.

\section{DUQUE DE CAXIAS}

Assim como os gestores do Rio de Janeiro (RJ), os entrevistados de Duque de Caxias (RJ) também apontaram a deficiência da formação e a aceitação do modelo de gestão alicerçado na saúde da família como fatores que dificultam o processo de gestão do trabalho. Estes implicam na desvalorização do vínculo com o usuário e limitam o desenvolvimento da $\mathrm{AB}$. "A faculdade ainda forma muito pouco para o SUS".

Outra dificuldade é vínculo com o paciente.

[...]. Isso, para alguns, é maravilhoso; para outros, é assustador [...] principalmente, para o médico. Acho que, na formação profissional, o enfermeiro é muito preparado para fazer vínculo, mas o médico ainda hoje é muito pouco preparado [...].

Assim, na perspectiva de aprimorar o atendimento dos usuários da ESF, realizado pelos profissionais de saúde, e ampliar o número de trabalhadores capacitados para atuar na $\mathrm{AB}$, o município vem adotando estratégias de qualificação da sua força de trabalho. "Oferecemos especialização em saúde da família [...] A gente tem incentivado o profissional a ter título de especialista."

No que tange à renumeração, os gestores mostram-se preocupados, sobretudo, em decorrência das investidas do município do Rio de Janeiro (RJ) para atrair profissionais, e cujo maior trunfo é o salário, que é superior ao oferecido por Duque de Caxias (RJ).

O salário é outro problema para o profissional. [...] O cara vai lá, dá um plantão de 24 horas e ganha $R \$ 7$ mil. Já aquele que está na saúde da família, naquela dedicação toda, com o vínculo com paciente, tem um salário que não chega a $R \$$ 5 mil. Então, isso desmotiva o profissional. 
Está havendo uma migração de profissionais, quando existe concurso público de outro município. [...] Nos dias de hoje, o que acontece é a remuneração baixa em relação à cidade do Rio de Janeiro (RJ).

A infraestrutura precária também foi citada como limitação ao processo de gestão, dado que a mesma dificulta o desenvolvimento das atividades e implica na insatisfação dos profissionais de saúde: "As unidades de PSF são puxadinhos, no meio de qualquer lugar, casa adaptada com teto caindo, sem estrutura nenhuma [...]”.

Nesses últimos aspectos, o município não tem conseguido efetivar estratégias de melhoria, seja no que se refere aos salários - em virtude da isonomia salarial, cujo aumento só pode se dar no âmbito do quadro geral da prefeitura -, seja com relação à infraestrutura, porque grande parte das Unidades de Saúde da Família existentes ou funcionam em locais alugados ou a prefeitura não possui, dos mesmos, os títulos de propriedade, de modo que não se podem promover reformas.

\section{Discussão}

Este artigo aborda as dificuldades relativas à gestão do trabalho da ESF percebidas pelos gestores de dois grandes municípios do Estado do Rio de Janeiro e as suas estratégias de enfrentamento. De um lado, a capital do Estado, que conjuga profissionais de vínculos e remuneração diferenciados: os estatutários e celetistas via OS. De outro, Duque de Caxias (RJ), que pratica a isonomia salarial para os servidores públicos em decorrência do nível de escolaridade.

Não obstante as diferenças dos agentes contratantes em relação aos vínculos e à remuneração, as dificuldades relativas à gestão do trabalho se assemelham nos dois municípios, segundo a percepção dos gestores. Dentre elas, foram citadas as limitações impostas por um processo de formação deficiente, que não prepara os profissionais para aceitarem e atuarem na perspectiva do modelo de gestão apregoado pelo SUS; a infraestrutura precária; e os vínculos e a remuneração praticados.

No que tange à formação de profissionais de saúde, é sabido que o sistema ainda carece de mão de obra com expertise e perfil para trabalhar na ESF, dado que, ainda hoje, o ensino ainda é influenciado pela concepção flexneriana e pela lógica de fragmentação dos conhecimentos (GONZÁLEZ; ALMEIDA, 2010).

Ainda pouco se valoriza a formação generalista dos profissionais de saúde, principalmente na medicina, ciência em que diversas especialidades se desenvolveram, provocando a fragmentação do cuidado e o desestímulo à obtenção de conhecimentos científicos comuns (FERREIRA; FIORINI; CRIVELARO, 2010).

Ademais, a hierarquização e a desintegração do conhecimento, bem como o ensino prático - que dicotomiza teoria e prática - ainda em pauta nas universidades, potencializam as resistências às mudanças, distanciando o profissional de saúde do serviço público e da $\mathrm{AB}$ (FERREIRA; FIORINI; CRIVELARO, 2010). Por consequência, reforça-se uma formação em saúde pautada em mecanicismo, biologismo, individualismo, especialização e curativismo, robustecendo as práticas do modelo flexneriano.

Diante disso, o grande desafio é reconhecer as deficiências da formação profissional em saúde e promover modificações no ensino que possam contribuir para um processo formativo, que releve as necessidades da população e que seja embasado no modelo de saúde que estrutura o SUS.

Nesse sentido, reforça-se a necessidade da criação de vínculos entre a universidade e a rede de saúde, para que os estudantes entrem em contato com a realidade da população e dos serviços de saúde pública e pratiquem efetivamente o exercício profissional na perspectiva do sistema de saúde. 
O estreitamento do vínculo entre a rede de saúde e as instituições de ensino técnico e superior promoveria uma transformação no processo formativo dos profissionais de saúde, arrefeceria o sentimento de isolamento percebido por muitos profissionais da ESF, bem como induziria um processo de educação continuada e permanente com vistas à atualização constante dos profissionais.

Não obstante os cursos de capacitação se mostrarem importantes e eficazes para o preparo do profissional na lógica do serviço, eles não são capazes, per si, de provocar mudanças no âmbito do ensino superior. Seria preciso maior articulação entre o ensino e o serviço para que este possa apresentar as deficiências da formação, as qualidades e as necessidades in loco que devem ser levadas em consideração no estabelecimento de um processo formativo alicerçado nas demandas do sistema de saúde e da população usuária (MOIMAZ ET AL., 2010).

Uma das dificuldades apontadas pelos gestores diz respeito à aceitação e ao entendimento do novo modelo de atenção orientado pela ESF. Encarar a transição do modelo de atenção flexneriano para o de vigilância em saúde configura-se como uma dificuldade enfrentada por trabalhadores, gestores e usuários. A resistência à mudança implica em obstáculo para o desenvolvimento e a consolidação da ESF, sobretudo em grandes municípios, onde ainda impera a rede de serviços terciária.

Quando comparados à $\mathrm{AB}$, os serviços terciários ainda são mais valorizados, apreciados como mais complexos e resolutivos, visto que concentram maior gama de equipamentos, técnicas de cuidado e atuam em diferentes situações que incorrem risco de vida. $\mathrm{E}$, neste sentido, valorizam-se muito os conhecimentos biológicos, patológicos e técnicos (SILVA JUNIOR; ALVES, 2007).

Nessa seara, estudos anteriores apontam alguns problemas para a consolidação da ESF no município de Duque de Caxias (RJ) - entre eles, a predominância das unidades básicas de saúde tradicionais com programas de saúde pública (MENDONÇA ET AL., 2006). Este município permanece mantendo o modelo tradicional de $\mathrm{AB}$ associado ao modelo da ESF. De tal modo, a ESF parece funcionar em caráter complementar e não em caráter substitutivo, visto que foi implantada e ainda se mantém como estrutura paralela à rede de saúde.

Ainda no que diz respeito ao modelo de atenção, os gestores de Duque de Caxias (RJ) ainda discorreram sobre outro problema enfrentado atualmente: o vínculo com o usuário, que intimida alguns profissionais, gerando, por consequência, a desaprovação, por parte da comunidade, para com esse profissional e, por vezes, com a equipe.

$\mathrm{O}$ vínculo com os usuários do serviço de saúde fomenta a efetividade das ações assistenciais, facilita a continuidade do acompanhamento terapêutico e favorece a participação ativa do usuário durante o cuidado, sendo, portanto, um dos pilares da ESF.

Muitas dificuldades vivenciadas por profissionais da ESF são atribuídas à organização do serviço, que, via de regra, é marcada por precariedade na infraestrutura. Todos os entrevistados realçaram a infraestrutura como sendo um fator importante a ser melhorado. O município do Rio de Janeiro (RJ) já vem empreendendo esforços neste sentido. Os gestores de Duque de Caxias (RJ), por outro lado, referem este como sendo um dos maiores motivos para a alta rotatividade de profissionais no município (MAGNAGO; PIERANTONI, 2014).

Pesquisa realizada por Ney e Rodrigues (2012) já havia identificado os problemas estruturais, as condições de trabalho inadequadas e insalubres das Unidades de Saúde da Família do município de Duque de Caxias (RJ).

A boa estruturação da ESF confere maior resolutividade no âmbito da $\mathrm{AB}$, reduzindo a procura de serviços de maior complexidade, o uso exagerado de medicamentos e de equipamentos de alta tecnologia, bem como favorecendo a reorganização da demanda. A precariedade da infraestrutura relatada pelos gestores dificulta a ampliação e o 
aprimoramento da rede básica de saúde via ESF, apontando a urgência de estratégias jurídicas que permitam a regularização das unidades de saúde de Duque de Caxias (RJ) e estejam habilitadas para a recepção de investimentos financeiros a serem alocados na melhoria dessa infraestrutura.

No que tange à remuneração e aos vínculos empregatícios, o cenário tem se apresentado melhor e cada vez mais promissor no município do Rio de Janeiro (RJ), segundo os gestores. Para eles, a responsabilidade deste feito, em parte, recai sobre a parceria estabelecida com as OS, que permitiu uma remuneração acima dos níveis salariais pagos pelo mercado de trabalho. Por outro lado, a contratação de profissionais de modo indireto gerou conflitos entre os servidores públicos municipais já atuantes na ESF e os profissionais contratados, uma vez que estes recebem salários superiores aos estatutários. Mesmo diante deste cenário, os benefícios da contratação indireta de recursos humanos parecem sobrepujar as desvantagens.

O valor dos salários representa hoje, para o Rio de Janeiro (RJ), uma estratégia eficaz de fixar os profissionais, ao passo que Duque de Caxias (RJ) tem enfrentado grandes dificuldades em manter a força de trabalho, especialmente médicos, com o salário ora praticado, computando, por exemplo, perda de profissionais para municípios vizinhos, como o próprio Rio de Janeiro (RJ).

O município de Duque de Caxias (RJ) pratica a isonomia salarial, de modo que os valores dos salários de enfermeiros e odontólogos ainda são superiores aos empregados pelo mercado de trabalho. Por outro lado, a isonomia salarial representa incômodo aos médicos, pois estes acreditam merecer um salário maior em virtude da alta responsabilidade que lhes é imputada. Adicionalmente, eles afirmam receber salários destoantes em relação às cargas horárias, às atribuições e aos valores pagos em outros municípios e, sobretudo, em relação a outras áreas de especialidade.

A gestão do trabalho na ESF dos municípios do Rio de Janeiro (RJ) e Duque de Caxias (RJ) apresenta diversos problemas que retardam o processo de consolidação do SUS. O cenário evidenciado por esta pesquisa não difere da realidade vivenciada por outros municípios.

Pesquisa realizada em quatro municípios com experiência consolidada de implementação da ESF apontou que a gestão do trabalho no âmbito da ESF tem buscado alternativas para a resolução de problemas relativos à consolidação do SUS municipal, tais como: regularização de vínculos, realização de concursos públicos, valorização da qualificação profissional, e remuneração adequada e recompensas para atuação em áreas de risCO (MENDONÇA ET AL., 2010).

Neste cenário, o avanço da ESF nos municípios de grande porte está condicionado a diversas mudanças no âmbito da gestão do trabalho, que perpassam não apenas a possibilidade de se obter profissionais de saúde habilitados a atuarem na perspectiva do SUS, mas também a oferta de postos de trabalho com salários competitivos no mercado de trabalho e que valorizem a dedicação do profissional ao serviço. E que, ainda, possa garantir a segurança e os insumos necessários ao desenvolvimento do trabalho.

Outro fator que imprime dificuldades à ESF é a falta de prestígio do médico de família por parte das entidades médicas e dos próprios colegas de profissão. Aliase, a isso, a hegemonia ainda hoje exercida pelos médicos e incitada pelas instituições de ensino, que se constituem como entraves para o desenvolvimento do trabalho multiprofissional e para o fortalecimento e o reconhecimento do papel assistencial de outros profissionais, obstando a possibilidade de ampliação da capacidade assistencial da ESF e a efetividade da Atenção Primária à Saúde.

Como nós críticos a serem desfeitos, e que foram citados pelos gestores, ainda têm-se o isolamento dos profissionais da saúde da família e o alto índice de rotatividade decorrente das dificuldades apresentadas neste artigo, bem como da localização das Unidades de Saúde da Família. 


\section{Conclusão}

Diante do exposto, evidencia-se que a gestão de RHS apresenta profundos contrastes entre os municípios pesquisados. No Rio de Janeiro (RJ), a contratação se dá por meio de organizações sociais que oferecem salários superiores à média praticada nos municípios vizinhos. Em Duque de Caxias (RJ), por sua vez, é praticada a isonomia salarial entre os profissionais de níveis equivalentes de escolaridade, o que se demonstrou como uma relevante iniciativa, rompendo, de certa forma, com o status quo nacional.

Diante desse cenário, foi possível perceber que o município do Rio de Janeiro (RJ), apesar dos problemas relatados, tem apresentado admiráveis mudanças, que demonstram uma postura de priorização da AB. Duque de Caxias (RJ) também caminha nessa direção, porém expõe maiores dificuldades em articular novas propostas que fomentem a priorização da ESF.

Em linhas gerais, o município do Rio de Janeiro (RJ) mostra um cenário mais atraente para os profissionais da ESF. A única vantagem de Duque de Caxias (RJ) evidenciada é a contratação direta pela prefeitura com vinculação estatutária, característica que se mostra mais interessante para os

\section{Referências}

BARDIN, L. Análise de conteúdo. Lisboa: Edições 70, 2002.

BRASIL. Secretaria de Políticas de Saúde. Análise de Reestruturação dos Modelos Assistenciais de Saúde em Grandes Cidades: padrões de custos e forma de financiamento. Brasília: Ministério da Saúde, 2002.

CAETANO, R.; DAIN, S. O Programa Saúde da Família e a reestruturação da atenção básica à saúde nos grandes centros urbanos: velhos problemas, novos desafios. odontólogos e enfermeiros. Por outro lado, ser estatutário em Duque de Caxias (RJ) significa dizer que o aumento salarial só existirá quando o aumento se der para todos os servidores municipais.

Ante os resultados evidenciados, sugere-se: (i) reestruturação dos currículos de graduação das áreas da saúde que permitam aos cursos adequar-se às necessidades do SUS e às do novo modelo de atenção alicerçado na $\mathrm{AB}$; (ii) estreitamento do vínculo entre as instituições de ensino superior e a rede de saúde que possibilitem a inserção de estudantes de graduação nas unidades de saúde, com vistas à ambientação na realidade social; (iii) ampliação de vagas em residências multiprofissionais e de uma política de valorização do trabalhador especializado em saúde da família; (iv) estabelecimento de fóruns de negociação com as representações das diversas categorias de saúde para a construção de uma concordância sobre as áreas de exercício profissional, no sentido de enfraquecer a hegemonia médica e ampliar as áreas de atuação dos demais profissionais, com vistas a ampliar a envergadura assistencial da ESF.

A consolidação da ESF nas metrópoles tem a potencialidade de despertar transformações no modelo de atenção que concretizem a saúde como direito social.

Physis, Rio de Janeiro, v. 12, n. 1, p. 11-23, jan/jun. 2002.

CAMPOS, F. E.; AGUIAR, R. A.; OLIVEIRA, V. B. O desafio da expansão do Programa de Saúde da Família nas grandes capitais brasileiras. Physis, Rio de Janeiro, v. 12 , n. 1, p.47-58, 2002.

ESCOREL, S. et al. O Programa de saúde da família e a construção de um novo modelo para a atenção básica no Brasil. Pan American Journal of Public Health, Washington, v. 21, n. 2/3, 2007, p. 164-176. 
FERREIRA, R. C.; FIORINI, V. M. L.; CRIVELARO, E. Formação profissional no SUS: o papel da atenção básica em saúde na perspectiva docente. Revista Brasileira de Educação Médica, Rio de Janeiro, v. 34, n. 2, p. 207-215, 2010.

GONZÁLEZ, A. D.; ALMEIDA, M. J. Movimentos de mudança na formação em saúde: da medicina comunitária às diretrizes curriculares. Physis, Rio de Janeiro, v. 20, n. 2, p. 551-570, 2010.

MAGNAGO, C.; PIERANTONI, C. R. A percepção de gestores dos municípios de Duque de Caxias e Rio de Janeiro quanto à rotatividade de profissionais na Estratégia Saúde da Família. Revista Cereus, Gurupi, v. 6, n. 1, p. 03-18, jan./abr. 2014.

MENDONÇA, M. H. M. M. et al. Desafios para gestão do trabalho a partir de experiências exitosas de expansão da Estratégia Saúde da Família. Ciência \&t Saúde Coletiva, Rio de Janeiro, v. 15, n. 5, p. 2355-2365, 2010.

MENDONÇA, M. H. M. M. et al. (Coord.). Relatório Estudo de Linha de Base - Projeto de Expansão da Estratégia de Saúde da Família no Município de Duque de Caxias, Estado do Rio de Janeiro. Rio de Janeiro: Escola Nacional de Saúde Pública, Fundação Oswaldo Cruz, 2006.
MOIMAZ, S. A. S. et al. Práticas de ensino-aprendizagem com base em cenários reais. Interface Comunicação, Saúde, Educação, São Paulo, v. 14, n. 32, p. 69-79, jan./mar. 2010.

NEY, M. S.; RODRIGUES, P. H. A. Fatores críticos para fixação do médico na Estratégia Saúde da Família. Physis, Rio de Janeiro, v. 22, n. 4, p. 1293-1311, 2012.

PIERANTONI, C. R.; VARELLA, T. C.; MONTEIRO, V. O. et al. Reconfigurando perfis profissionais: a especialização em saúde da família. In: PIERANTONI, C. R.; VIANA, A. L. D. (Org.). Educação e Saúde. São Paulo: Hucitec, 2010. p. 224-39.

SILVA JÚNIOR, A. G.; ALVES, C. A. Modelos Assistenciais em Saúde: desafios e perspectivas. In: MOROSINI, M. V. G. C. Modelos de atenção e a saúde da família. Rio de Janeiro: EPSJV/Fiocruz, 2007.

VIANA, A. L. D.; DAL POZ, M. R. A Reforma do Sistema de Saúde no Brasil e o Programa de Saúde da Família. Physis, Rio de Janeiro, v. 12, supl., p. 255-264, 2005.

Recebido para publicação em abril de 2014

Versão final em novembro de 2014

Conflito de interesse: inexistente

Suporte financeiro: não houve 